\title{
Consumo de antimicrobianos parenterales en diferentes hospitales de Chile durante el año 2005
}

\author{
Alberto Fica C., Angela Cabello M., Chrystal Juliet L., Priscilla Prado D. y Luis Bavestrello F. en representación del \\ Comité Consultivo de Antimicrobianos de la Sociedad Chilena de Infectología
}

\section{Intravenous antimicrobial use among different hospital in Chile during 2005}

Intravenous antimicrobial consumption has not been evaluated previously in Chile. In order to know this consumption (in DDD per 100 bed days), associated factors and antimicrobial control systems across the country, a questionnaire was sent to evaluate these features during 2005. A total of 29 public hospitals and private clinics answered this poll, 20 belonging to the public health system (69\%). Only $48.1 \%$ declared to have an independent antimicrobial committee and 17.2\% allowed unrestricted antimicrobial use. Glycopeptides and carbapenems were the most regulated compounds (75.9 and $82.8 \%$, respectively). Antimicrobial controls systems were more frequently declared among public hospitals and only non-public hospitals permitted free use of antimicrobials. Global consumption reached 59.98 DDD per 100 bed-days, with beta-lactams representing $74.3 \%$ of this consume (44.57 DDD per 100), and cephalosporins 43\% (25.78 DDD per 100). Chloramphenicol, penicillin $\mathrm{G}$ and cloxacillin use was significantly higher among public hospitals. The opposite was observed for imipenem-cilastatin, linezolid, cefuroxime and caspofungin with higher consumes observed among nonpublic hospitals. In a multivariate analysis, increased cefazolin use was independently associated with sites allowing unrestricted use, and ciprofloxacin consumption with non-public hospitals. Institutions with decreased susceptibility to imipenem-cilastatin among non-fermentative gram negative bacilli showed a higher use of this compound and linezolid consumption paralleled vancomycin-resistant enterococci prevalence. It is necessary to reinforce governmental regulations about antimicrobial use issued during 1999.

Key words: Antimicrobial, intravenous, consumption, rational use, resistance.

Palabras clave: Antimicrobianos, intravenoso, consumo, uso racional, resistencia.

\section{Introducción}

$\mathrm{E}$ 1 uso de antimicrobianos está asociado al riesgo de una indicación inapropiada, a la selección o inducción de cepas resistentes y al aumento innecesario de los costos.

El consumo de antimicrobianos puede ser abordado desde diferentes perspectivas. Una de ellas, la evaluación del consumo en Dosis Diaria Definida (DDD) por 100 días camas, que refleja cuántos pacientes cada 100 están recibiendo diariamente un tratamiento específico, ha sido ampliamente utilizada y ha permitido comparar diferentes escenarios, tendencias, asociación con resistencia y en forma más importante, el efecto de diferentes medidas de intervención ${ }^{1-5}$.

Al menos cuatro trabajos sobre consumo de antimicrobianos se han desarrollado previamente en Chile ${ }^{6-9}$. Tres de estos estudios se han enfocado al consumo comunitario de estos compuestos y sólo uno al consumo hospitalario pero limitado a una sola institución en
Santiago. En este estudio se logró demostrar un aumento de la resistencia antimicrobiana en forma posterior al incremento de uso de ampicilina y cefazolina?.

Aumentos en el consumo de antimicrobianos han sido asociados a un aumento en la resistencia antimicrobiana comunitaria y hospitalaria y este fenómeno ha sido descrito tanto en hospitales como en comunidades, países y continentes, abarcando microorganismos grampositivos y gramnegativos ${ }^{3,10-17}$.

El consumo de antimicrobianos en los hospitales es una gran fuerza motriz en la selección y propagación de bacterias resistentes y multiresistentes; no obstante, no se dispone de información sobre la magnitud de este consumo en los hospitales en Chile y tampoco si existen variaciones regionales $\mathrm{u}$ otros factores involucrados. Asimismo, se desconoce si se encuentran en práctica, a nivel local, las regulaciones sobre el control de estos compuestos que rigen desde el año 1999 para todo el país. El tener información sobre este consumo es de vital importancia para reforzar estas medidas
Hospital Clínico Universidad de Chile, Santiago (AFC) Hospital Gustavo Fricke, Viña del Mar (ACM) Hospital Del Salvador, Santiago $(C L)$ Hospital Padre Hurtado, Santiago (PPD) Clínica Reñaca, Viña del Mar (LBF)

Recibido: 17 enero 2008 Aceptado: 11 agosto 2008

Correspondencia a: Alberto Fica Cubillos afica@redclinicauchile.cl 
regulatorias o, incluso, diseñar nuevos programas de intervención institucional o nacional. Por ello, el Comité Consultivo de Antimicrobianos de la Sociedad Chilena de Infectología decidió efectuar un estudio para conocer cuáles son los patrones de este consumo en los hospitales, los potenciales factores que la explican y su posible asociación con resistencia antimicrobiana.

\section{Método}

Para averiguar los consumos de diferentes compuestos antimicrobianos inyectables se elaboró una encuesta que fue dirigida desde la Sociedad Chilena de Infectología, al Director de cada hospital o clínica en todo el país. Se invitó a participar de la encuesta a diversas instituciones públicas y privadas.

Muestra de análisis. Se consideraron hospitales públicos, centros nacionales de referencia, hospitales universitarios, hospitales de las fuerzas armadas (FF.AA.) y algunas clínicas privadas.

Hospitales públicos y centros de referencia. Se consideraron todos los hospitales públicos que sirven como institución de referencia para cada uno de los distintos Servicios de Salud del país y que corresponden a hospitales de tipo 1 (mayor complejidad). Además se incluyeron dos hospitales públicos que actúan como centro nacional de enfermedades respiratorias y de neurocirugía, respectivamente y un hospital dedicado a la atención de patología de urgencia.

Hospitales universitarios y de fuerzas armadas. La encuesta consideró la inclusión de los dos únicos hospitales universitarios existentes en el país, ambos en la Región Metropolitana (RM) y de los cuatro principales hospitales de las FF.AA. (Militar, Fuerza Aérea y Carabineros en la RM y Naval en la Región de Valparaíso). Además, se incluyó un hospital para personal jubilado y familiares de Carabineros de Chile, también ubicado en la RM.

Clínicas privadas. A diferencia de la muestra para hospitales públicos, universitarios y de FF.AA. donde se consideró la totalidad de los hospitales de alta complejidad, en el caso de las clínicas privadas sólo se consideró una muestra exploratoria para los centros presentes en la RM.

Hospitales no considerados. No se incluyeron en este estudio hospitales públicos pediátricos, ni hospitales públicos de menor complejidad ubicados en la $\mathrm{RM} \mathrm{u}$ otras regiones.

Contenidos de la encuesta. Las preguntas incluidas iban dirigidas a describir cuatro áreas:

- Datos generales del hospital o clínica tales como número de camas, índice ocupacional, tipo de servicios, existencia de UCI de adultos y de niños, etc.
La información sobre la complejidad de los hospitales se obtuvo del Departamento de Estadísticas e Información de Salud del Ministerio de Salud.

- Organización para lograr el uso racional de antimicrobianos, con preguntas dirigidas a averiguar la existencia de un Comité de Farmacia, un Comité de Antimicrobianos (independiente al anterior), la existencia de un arsenal farmacológico, programa de restricción o visado al uso de antimicrobianos, controles específicos sobre algunos compuestos tales como carbapenémicos, glicopéptidos, amikacina, quinolonas o cefalosporinas de $3^{\mathrm{a}}$ generación. Estas preguntas también iban dirigidas a indagar si se efectuaba auditoría de recetas y si el informe del antibiograma estaba restringido a los compuestos presentes en el arsenal y era decidido por el propio laboratorio de microbiología.

- Consumo de antimicrobianos. Se consideraron todos los compuestos parenterales, tanto convencionales como aquellos de reciente incorporación. En los antibacterianos se incluyeron $\beta$-lactámicos y derivados, quinolonas, glicopéptidos, aminoglucósidos, cloranfenicol, metronidazol, colistín y linezolid. Se analizó también el consumo de anfotericina B, caspofungina y fluconazol, así como el uso de aciclovir y ganciclovir. El consumo se calculó según las recomendaciones de la OMS, en base a las DDD por 100 días camas ${ }^{18}$.

- En la parte final de la encuesta se pidió a los centros participantes informar sobre la frecuencia de susceptibilidad antimicrobiana ante diferentes compuestos, precisando si los datos incluían pacientes de UCI y/o muestras de origen comunitario.

Análisis estadístico. Debido al bajo tamaño de la muestra, los análisis estadísticos fueron desarrollados con pruebas no paramétricas. En el caso de variables categóricas, se utilizaron pruebas de $\chi^{2}$ o Fisher y en el caso de variables continuas el test de KolmogorovSmirnov para muestras independientes. Se utilizó el coeficiente de correlación de Spearman para comparar dos variables continuas y se consideró un nivel de significación con un valor $\mathrm{p}<0,05$. En caso de diferencias de consumo para un mismo compuesto con diferentes variables categóricas, se aplicó una prueba de regresión lineal múltiple como análisis multivariado.

\section{Resultados}

Características generales. Un total de 29 hospitales o clínicas respondieron la encuesta, 20 de ellos de tipo público $(69 \%)$, cinco de FF.AA. $(17,2 \%)$, tres de carácter privado $(10,3 \%)$ y uno universitario $(3,4 \%)$. El 
rango de camas disponibles era de 97 a 704 y el índice ocupacional varió entre 60 y $89 \%$. Un 86,2\% (n: 25) declaró contar con camas de intensivo de adultos y otro $58,6 \%$ (n: 17 ) con camas de intensivo pediátricas.

Del total de 182 hospitales públicos (tipos 1 al 4, de mayor a menor complejidad) existentes en el país, 20 fueron incluidos en esta encuesta (11\%). Se incluyeron específicamente 11 de los 23 hospitales tipo 1 $(47,8 \%), 8$ de los 37 del tipo $2(21,6 \%)$ y 1 de los 23 de tipo $3(4,3 \%)$.

Control sobre el uso de antimicrobianos. La existencia de un Comité de Farmacia, de un arsenal farmacológico definido, de un listado restringido y de un sistema de visado fueron declarados por casi la totalidad de los hospitales encuestados. De la misma manera, en todos los casos, los compuestos incluidos en el antibiograma eran decididos por el laboratorio. (Tabla 1).

Las respuestas fueron variables respecto a la existencia de un Comité de Antimicrobianos (sólo presente en $48,1 \%$ ), si se utilizaban antimicrobianos no disponibles en el arsenal, a la auditoría de recetas y a la existencia de programas de control sobre carbapenémicos, glicopéptidos, amikacina, cefalosporinas de $3^{\text {a }}$ generación, quinolonas y amikacina. Sin embargo, los carbapenémicos y glicopéptidos fueron los compuestos más supervisados en esta muestra $(82,8$ y $75,9 \%$, respectivamente). De la misma manera, las restricciones al informe de susceptibilidad por parte del laboratorio y el estudio de moléculas fuera del arsenal, fueron declaradas en grado variable (Tabla 1).

Los hospitales encuestados declararon, también en grado variable, un uso restringido para diferentes compuestos (Tabla 2). Concordante con el concepto de compuestos de primera línea, ampicilina, bencilpenicilina $\mathrm{G}$ y penicilina benzatina, cloxacilina y cloranfenicol, fueron los únicos compuestos que no tenían restricción para su uso (Tabla 2).

Entre los $\beta$-lactámicos, destacó el uso restringido sobre cefalosporinas de primera y segunda generación y los altos porcentajes de restricción ante compuestos carbapenémicos, sulbactam/cefoperazona y piperacilina/tazobactam. Este fenómeno se repitió ante vancomicina. Notoria es también la restricción observada sobre quinolonas y clindamicina $(>50 \%)$. Los compuestos antifúngicos y antivirales parenterales también aparecen con uso restringido (Tabla 2).

Los programas de control fueron significativamente más frecuentes en los hospitales públicos que en los no públicos (incluyendo bajo este último concepto los hospitales de las FF.AA., clínicas privadas y establecimientos universitarios), excepto para el caso de amikacina (Tabla 3). El control sobre cefalosporinas de $3^{\text {a }}$ generación fue más frecuente fuera de la RM que en
Tabla 1. Control sobre el uso de diferentes antimicrobianos en 29 hospitales en Chile durante el 2005

\begin{tabular}{|c|c|c|}
\hline Variable (n) & $\mathbf{n}$ & $\% *$ \\
\hline Existe un Comité de Farmacia (29) & 28 & 96,6 \\
\hline Existe un Comité de Antimicrobianos (27) & 13 & 48,1 \\
\hline Existe un arsenal farmacológico definido (28) & 27 & 96,4 \\
\hline Todos los antimicrobianos están incluidos en el arsenal (29) & 16 & 55,2 \\
\hline Existe listado de antimicrobianos restringidos (26) & 24 & 92,3 \\
\hline Existe un sistema de visado (26) & 24 & 92,3 \\
\hline Control sobre carbapenémicos (29) & 24 & 82,8 \\
\hline Control sobre glicopéptidos (29) & 22 & 75,9 \\
\hline Control sobre cefalosporinas de $3^{a}$ generación (28) & 18 & 64,3 \\
\hline Control sobre quinolonas (27) & 18 & 66,7 \\
\hline Control sobre amikacina (27) & 7 & 25,9 \\
\hline Auditoría de recetas (28) & 12 & 42,9 \\
\hline Laboratorio decide el antibiograma (26) & 26 & 100 \\
\hline Laboratorio informa sólo el arsenal (26) & 14 & 53,8 \\
\hline Laboratorio restringe el informe de susceptibilidad (27) & 19 & 70,4 \\
\hline Todos los antimicrobianos se pueden indicar sin restricción (29) & 5 & 17,2 \\
\hline
\end{tabular}

ésta (11 de 12 versus siete de $16 ; \mathrm{p}=0,01)$ sin encontrarse diferencias significativas para otros compuestos.

Sólo hospitales no públicos permiten el uso universal de antimicrobianos sin restricción. Cinco de nueve hospitales no públicos permitían el uso libre sin restricciones $(55,6 \%)$ versus $0 \%$ de los hospitales públi$\cos (\mathrm{p}=0,001)$.

Consumo de antimicrobianos. Por cada 100 días camas ocupadas, ceftriaxona fue el antimicrobiano parenteral más consumido en los 29 hospitales encuestados durante el año 2005 (Tabla 4 y Figura 1), representando cada día casi 18 tratamientos por cada 100 camas ocupadas. El consumo fue secundado en forma decreciente por cloxacilina, bencilpenicilina $G$, cefazolina, metronidazol, gentamicina, ampicilina, vancomicina y clindamicina. Estos nueve compuestos representan más de 50 tratamientos diarios por cada 100 camas ocupadas $(51,33)$.

El consumo conjunto de todos los compuestos antibacterianos representó 59,98 DDD por cada 100 días camas ocupadas. El consumo de todos los $\beta$-lactámicos alcanzó un valor de 44,57 y el de cefalosporinas un valor global de 25,78 DDD por cada 100 días camas ocupadas. Estas cifras representan 74,3 y $43 \%$, respectivamente, de la suma total de tratamientos antibacterianos por 100 días camas. En tanto, el consumo 
Tabla 2. Uso restringido de compuestos específicos según declaración

\begin{tabular}{|c|c|c|}
\hline Antimicrobiano & $\begin{array}{c}\text { Hospitales que especifican } \\
\text { uso libre o restringido } \\
\text { n }\end{array}$ & $\begin{array}{r}\% \text { con uso } \\
\text { restringido }\end{array}$ \\
\hline \multicolumn{3}{|l|}{ Beta-lactámicos } \\
\hline Ampicilina & 20 & 0,0 \\
\hline Cloxacilina & 21 & 0,0 \\
\hline Penicilina G & 20 & 0,0 \\
\hline Penicilina benzatina & 10 & 0,0 \\
\hline Cefazolina & 21 & 42,8 \\
\hline Cefuroxima & 7 & 42,8 \\
\hline Cefotaxima & 18 & 61,1 \\
\hline Ceftriaxona & 21 & 57,1 \\
\hline Ceftazidima & 22 & 63,6 \\
\hline Sulbactam/ampicilina & 16 & 68,8 \\
\hline Sulbactam/cefoperazona & 20 & 85,0 \\
\hline Piperacilina/tazobactam & 8 & 87,5 \\
\hline Imipenem-cilastatina & 21 & 85,7 \\
\hline Meropenem & 16 & 87,5 \\
\hline Ertapenem & 12 & 91,7 \\
\hline \multicolumn{3}{|l|}{ Otros compuestos } \\
\hline Cloranfenicol & 20 & 0,0 \\
\hline Metronidazol & 20 & 35,0 \\
\hline Clindamicina & 21 & 57,1 \\
\hline Ciprofloxacina & 19 & 68,4 \\
\hline Vancomicina & 22 & 81,8 \\
\hline Colistin & 4 & 100,0 \\
\hline Linezolid & 6 & 83,3 \\
\hline Cotrimoxazol & 17 & 47,0 \\
\hline \multicolumn{3}{|l|}{ Antifúngicos } \\
\hline Anfotericina B & 19 & 89,5 \\
\hline Fluconazol & 20 & 70,0 \\
\hline Caspofungina & 6 & 83,3 \\
\hline \multicolumn{3}{|l|}{ Antivirales } \\
\hline Aciclovir & 19 & 68,4 \\
\hline Ganciclovir & 10 & 90,0 \\
\hline
\end{tabular}

Tabla 3. Distribución de programas de control de diferentes compuestos según tipo de hospital

\begin{tabular}{|lccc|} 
& \multicolumn{4}{c|}{ Tipo de hospital } \\
Control sobre & Público $(\mathbf{n} / \mathbf{N})$ & No público $(\mathbf{n} / \mathbf{N})$ & P \\
\hline Carbapenémicos & $19 / 20$ & $5 / 9$ & 0,02 \\
Vancomicina & $18 / 20$ & $4 / 9$ & 0,02 \\
\hline Cefalosporinas $3^{a}$ G & $16 / 19$ & $2 / 9$ & $<0,05$ \\
Quinolonas & $16 / 18$ & $2 / 9$ & 0,001 \\
Amikacina & $7 / 18$ & $0 / 9$ & $<0,59$ \\
\hline
\end{tabular}

específico de ceftriaxona y cefazolina alcanzó a 39,07\% del consumo total.

En la Tabla 4 se puede apreciar también que para productos similares, imipenem-cilastatina fue consumido tres veces más frecuentemente que meropenem $(0,75$ vs 0,25$)$ y que estas diferencias eran mayores en el caso de sulbactam/cefoperazona respecto a piperacilina/tazobactam $(0,54$ vs 0,07$)$. De la misma manera, metronidazol fue utilizado más frecuentemente que cloranfenicol.

En la Tabla 5 se muestran los consumos observados para compuestos antifúngicos y antivirales parenterales, observándose un predominio de fluconazol y ganciclovir, respectivamente. Sin embargo, las magnitudes de estos consumos eran bajos respecto a los compuestos antibacterianos.

Factores asociados al consumo de compuestos antibacterianos. Los rangos de consumo variaron en forma importante para cada uno de los compuestos analizados, indicando la posible participación de algunos factores asociados.

Se exploraron diferentes componentes incluidos en la encuesta, encontrando en un análisis univariado, asociaciones para algunos compuestos con el tipo de hospitales, su ubicación geográfica (dentro o fuera de la $\mathrm{RM})$, índice ocupacional $(\leq \mathrm{o}>0,75)$, existencia de UCI de adultos y aplicación o no de restricción (Tabla 6).

El consumo de cloranfenicol, bencilpenicilina G y cloxacilina fue significativamente más alto en los hospitales públicos y los consumos de cefazolina, imipenem-cilastatina, ciprofloxacina, linezolid, cefuroxima, fluconazol y caspofungina, fueron más elevados en los hospitales no públicos (Tabla 6).

Los consumos de gentamicina y cloranfenicol fueron más elevados en hospitales fuera de la RM. En contraste, el consumo de cefazolina, vancomicina y ganciclovir fue más alto en la RM.

Los hospitales con menor índice ocupacional presentaron un consumo más alto de cefazolina, cotrimoxazol, ciprofloxacina y fluconazol y un consumo menor de cloxacilina respecto de los hospitales con índice ocupacional mayor (Tabla 6).

Los hospitales que tienen UCI de adultos, presentaron un mayor consumo de ciprofloxacina respecto a los que no tienen esta unidad (Tabla 6).

Finalmente, el consumo de cefazolina y de ciprofloxacina fue mayor en aquellos hospitales o clínicas que permitían el uso de antimicrobianos sin ningún tipo de restricción (Tabla 6).

La existencia de un Comité de Antimicrobianos, de un sistema de visado, la auditoría de recetas o la restricción del informe de susceptibilidad por parte del laboratorio, no estuvieron asociados a diferencias de consumo entre diferentes hospitales. 
Un análisis multivariado mediante regresión lineal múltiple asoció las diferencias observadas en el consumo de cefazolina al uso libre de antimicrobianos sin restricción y no al tipo de hospital, región o índice ocupacional $(\mathrm{p}=0,001)$.

En el caso de ciprofloxacina, las diferencias son explicadas por el tipo de hospital (público/privado) y no por diferencias en el índice ocupacional, existencia de UCI adultos o uso permisivo sin restricciones para los diferentes antimicrobianos $(\mathrm{p}<0,001)$.

De la misma manera, las diferencias observadas en el consumo de fluconazol, son explicadas por el tipo de hospital (público/privado) y no por diferencias en el índice ocupacional $(\mathrm{p}<0,001)$.

Porcentajes de susceptibilidad a diferentes antimicrobianos. Para evitar sesgos, la información fue separada entre aquellos hospitales que reportaron sólo información de aislados asociados a infecciones intrahospitalarias (IIH), del resto de los hospitales que enviaron información conjunta.

En la Tabla 7 se detallan los datos para aquellos hospitales que enviaron sólo información de IIH. Los porcentajes de susceptibilidad resultaron reducidos para cocáceas grampositivas y bacilos gramnegativos típicamente ligados a infecciones nosocomiales y que incluyen cloxacilina en el caso de $S$. aureus, ceftazidima y ciprofloxacina en E. coli y K. pneumoniae, sulbactam/ cefoperazona en $A$. baumannii e imipenem-cilastatina en $P$. aeruginosa (Tabla 7). El bajo número de hospitales con información sobre IIH impidió explorar factores ligados a estos resultados.

Cuando se analizó la información global de susceptibilidad, se identificó un mayor porcentaje de suscep-
Tabla 4. Consumo en DDD por 100 días camas ocupadas de compuestos antibacterianos parenterales en 29 hospitales de Chile. Año 2005

\begin{tabular}{lcccc} 
Compuesto & Mínimo & Máximo & Promedio & DE \\
\hline Ceftriaxona & 2,27 & 78,09 & 17,89 & 13,77 \\
\hline Cloxacilina & 0,00 & 59,70 & 8,71 & 10,59 \\
\hline Penicilina G & 0,29 & 27,45 & 5,62 & 5,29 \\
\hline Cefazolina & 0,00 & 26,12 & 5,55 & 5,26 \\
\hline Metronidazol & 0,00 & 9,08 & 4,66 & 2,67 \\
\hline Gentamicina & 0,14 & 7,94 & 2,84 & 2,34 \\
\hline Ampicilina & 0,00 & 6,29 & 2,18 & 1,74 \\
\hline Vancomicina & 0,23 & 4,86 & 1,97 & 1,42 \\
\hline Clindamicina & 0,41 & 4,14 & 1,91 & 1,03 \\
\hline Cloranfenicol & 0,00 & 6,49 & 1,45 & 1,54 \\
\hline Amikacina & 0,00 & 6,94 & 1,42 & 1,25 \\
\hline Cefotaxima & 0,00 & 8,78 & 1,15 & 1,89 \\
\hline Penicilina benzatina & 0,00 & 3,66 & 0,82 & 1,00 \\
\hline Imipenem-cilastatina & 0,00 & 2,67 & 0,72 & 0,70 \\
\hline Ciprofloxacina & 0,00 & 2,79 & 0,68 & 0,86 \\
\hline Sulbactam/cefoperazona & 0,00 & 3,38 & 0,54 & 0,87 \\
\hline Ceftazidima & 0,00 & 1,22 & 0,45 & 0,29 \\
\hline Cotrimoxazol & 0,00 & 3,69 & 0,45 & 0,89 \\
\hline Meropenem & 0,00 & 3,53 & 0,25 & 0,65 \\
\hline Ampicilina/sulbactam & 0,00 & 1,10 & 0,18 & 0,24 \\
\hline Ertapenem & 0,00 & 1,23 & 0,13 & 0,29 \\
\hline Cefuroxima & 0,00 & 0,67 & 0,11 & 0,21 \\
\hline Cefepime & 0,00 & 2,45 & 0,09 & 0,45 \\
\hline Amoxicilina/ác. clavulánico & 0,00 & 1,27 & 0,07 & 0,25 \\
\hline Piperacilina/tazobactam & 0,00 & 0,67 & 0,07 & 0,16 \\
\hline Amoxicilina/sulbactam & 0,00 & 0,70 & 0,04 & 0,14 \\
\hline Colistín & 0,00 & 0,25 & 0,02 & 0,06 \\
\hline Linezolid & 0,14 & 0,01 & 0,03 \\
\hline DE: desviación estándar & & & & \\
\hline & & & & \\
\hline & 0,00 & 0,15 & \\
\hline
\end{tabular}

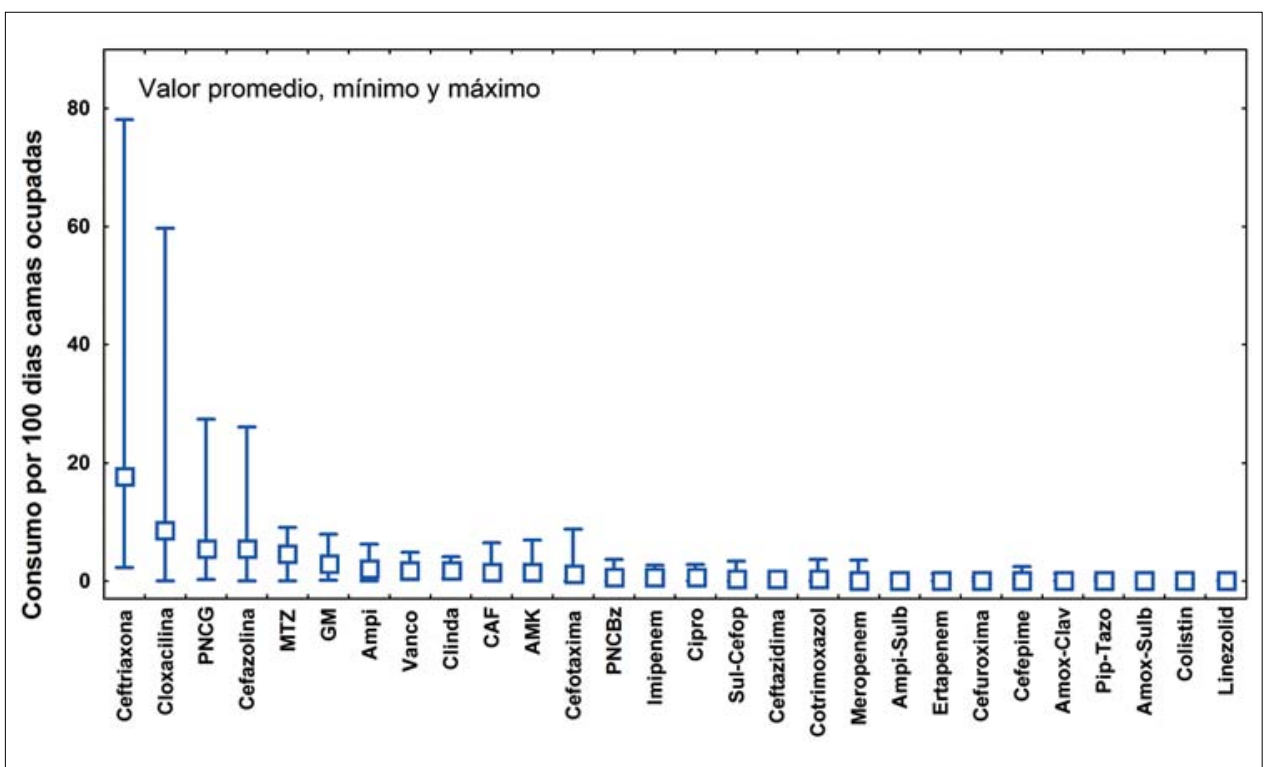

Figura 1. Consumo de compuestos antibacterianos en 29 hospitales de Chile durante el 2005. PNCG: penicilina G. MTZ: metronidazol. GM: gentamicina. Ampl.: ampicilina. Vanco: vancomicina. Clinda: clindamicina. CAF: cloranfenicol. AMK: amikacina. PNCBz: penicilina benzatina. Cipro: ciprofloxacina. Sul-cefop: sulbactam/cefoperazona. Ampi-sulb: ampicilina/sulbactam. Amox-clav: amoxicilina/ác. clavulánico. Pip-Tazo: piperacilina/tazobactam. Amoxisulb: amoxicilina/sulbactam. 
Tabla 5. Consumo en DDD por 100 días camas ocupadas de compuestos antifúngicos y antivirales parenterales en 29 hospitales de Chile. Año 2005

\begin{tabular}{lcccc|}
$\begin{array}{l}\text { Grupo de acción } \mathbf{y} \\
\text { compuesto }\end{array}$ & Mínimo & Máximo & Promedio & DE \\
\hline Antifúngicos & & & & \\
Fluconazol & 0,00 & 1,50 & 0,24 & 0,38 \\
Anfotericina B deoxicolato & 0,00 & 0,92 & 0,14 & 0,22 \\
Caspofungina & 0,00 & 1,14 & 0,06 & 0,22 \\
Antivirales & & & & \\
Ganciclovir & 0,00 & 0,45 & 0,05 & 0,10 \\
Aciclovir & 0,00 & 0,16 & 0,03 & 0,04 \\
\hline
\end{tabular}

\section{Tabla 6. Consumo en DDD por 100 días camas ocupadas según variables} de agrupación para compuestos con diferencias significativas. Análisis univariado

\begin{tabular}{|c|c|c|c|}
\hline Compuesto & $\begin{array}{l}\text { Promedio ( } \pm \text { SD) } \\
\text { Hospitales públicos }\end{array}$ & $\begin{array}{l}\text { Promedio }( \pm \text { SD) } \\
\text { Otros hospitales }\end{array}$ & $\mathbf{P}$ \\
\hline Cefazolina & $3,18 \pm 1,99$ & $10,82 \pm 6,49$ & $<0,001$ \\
\hline Imipenem-cilastatina & $0,48 \pm 0,45$ & $1,23 \pm 0,89$ & 0,005 \\
\hline Cloranfenicol & $1,78 \pm 1,62$ & $0,69 \pm 1,03$ & $<0,05$ \\
\hline Penicilina G & $6,89 \pm 5,89$ & $2,81 \pm 1,66$ & $<0,05$ \\
\hline Cloxacilina & $10,80 \pm 12,18$ & $4,06 \pm 2,06$ & $<0,01$ \\
\hline Ciprofloxacina & $0,19 \pm 0,16$ & $1,75 \pm 0,79$ & $<0,001$ \\
\hline Linezolid & $0,003 \pm 0,008$ & $0,027 \pm 0,047$ & $<0,05$ \\
\hline Cefuroxima & $0,02 \pm 0,07$ & $0,30 \pm 0,27$ & $<0,05$ \\
\hline Fluconazol & $0,08 \pm 0,09$ & $0,60 \pm 0,51$ & $<0,01$ \\
\hline \multirow[t]{2}{*}{ Caspofungina } & $0,0005 \pm 0,002$ & $0,19 \pm 0,37$ & $<0,01$ \\
\hline & $\begin{array}{l}\text { Hospitales Región } \\
\text { Metropolitana }\end{array}$ & $\begin{array}{l}\text { Hospitales en } \\
\text { otra región }\end{array}$ & \\
\hline Gentamicina & $2,29 \pm 2,38$ & $3,62 \pm 2,13$ & $<0,05$ \\
\hline Cefazolina & $7,20 \pm 6,34$ & $3,21 \pm 1,32$ & $<0,05$ \\
\hline Cloranfenicol & $1,16 \pm 1,48$ & $1,84 \pm 1,58$ & $<0,01$ \\
\hline Vancomicina & $2,69 \pm 1,39$ & $0,94 \pm 0,57$ & $<0,01$ \\
\hline \multirow[t]{2}{*}{ Ganciclovir } & $0,08 \pm 0,12$ & $0,006 \pm 0,02$ & $<0,01$ \\
\hline & $\begin{array}{c}\text { Índice ocupacional } \\
\leq 0,75\end{array}$ & $\begin{array}{c}\text { Índice ocupacional } \\
>0,75\end{array}$ & \\
\hline Cefazolina & $9,53 \pm 6,47$ & $3,12 \pm 2,10$ & $<0,01$ \\
\hline Cotrimoxazol & $1,02 \pm 1,27$ & $0,09 \pm 0,09$ & $<0,05$ \\
\hline Cloxacilina & $4,93 \pm 2,11$ & $11,02 \pm 12,92$ & $<0,01$ \\
\hline Ciprofloxacina & $1,36 \pm 1,04$ & $0,25 \pm 0,27$ & $<0,01$ \\
\hline \multirow[t]{2}{*}{ Fluconazol } & $0,46 \pm 0,51$ & $0,10 \pm 0,15$ & $<0,05$ \\
\hline & UCI adultos & Sin UCI adultos & \\
\hline \multirow[t]{2}{*}{ Ciprofloxacina } & $0,78 \pm 0,88$ & $0,02 \pm 0,2$ & $<0,01$ \\
\hline & $\begin{array}{c}\text { Uso libre de } \\
\text { cualquier } \\
\text { antimicrobiano }\end{array}$ & $\begin{array}{l}\text { Restricción para } \\
\text { algunos } \\
\text { antimicrobianos }\end{array}$ & \\
\hline Cefazolina & $14,41 \pm 6,68$ & $3,70 \pm 2,30$ & $<0,001$ \\
\hline Ciprofloxacina & $1,86 \pm 0,94$ & $0,43 \pm 0,60$ & $<0,05$ \\
\hline
\end{tabular}

tibilidad a vancomicina en Enterococcus sp y a imipenem-cilastatina en $P$. aeruginosa, en hospitales públicos respecto a otro tipo de hospitales (Tabla 8).

La presencia o ausencia de un programa de control sobre carbapenémicos, vancomicina, cefalosporinas, quinolonas o amikacina, no se asoció a diferencias en porcentajes de susceptibilidad a estos compuestos a través de diferentes hospitales (datos no mostrados).

Las instituciones que tuvieron un mayor consumo de imipenem-cilastatina tuvieron menor susceptibilidad a este mismo compuesto, tanto en $P$. aeruginosa como en A. baumannii. En contraste, el consumo de colistín resultó mayor en aquellos establecimientos con menor susceptibilidad a imipenem-cilastatina para estas mismas dos especies. Por su parte, el consumo de linezolid fue mayor en aquellos sitios que presentaron una menor susceptibilidad a vancomicina entre los aislados de Enterococcus sp (Tabla 9).

\section{Discusión}

El estudio del consumo de antimicrobianos ha demostrado ser importante para identificar problemas epidemiológicos, para desarrollar políticas de intervención y monitorizar conductas terapéuticas. Por ejemplo, estudios previos en Chile lograron detectar hacia 1983-84, el gran volumen de unidades adquiridas sin receta médica y el sub-tratamiento de diferentes condiciones mórbidas ${ }^{8}$. Por otra parte, Bavestrello y cols, informaron hacia el año 2000, del impacto que tuvo la introducción de la exigencia de la receta médica para la venta de antimicrobianos en farmacias, con una caída drástica en el consumo poblacional ${ }^{7}$.

Varios hallazgos importantes fueron identificados en este trabajo. En primer lugar, la norma del Ministerio de Salud de Chile, vigente desde el año 1999 (circular $4 \mathrm{C} / 32$ ) y que establece la necesidad de contar con un programa de uso racional de estos compuestos, parece haber tenido una adherencia incompleta en los hospitales y clínicas de esta muestra. Respaldan este concepto el hecho de que $17,2 \%$ de los lugares encuestados aún permiten el uso libre sin restricciones de todos los antimicrobianos y que otro $25 \%$ no ejerce control sobre el uso de glicopéptidos, molécula específicamente comentada en la norma. No obstante, el control sobre el uso de estas herramientas terapéuticas es más frecuente en el sistema público, demostrando una mejor preparación y ejecución de esta indicación.

Un segundo hallazgo importante es el amplio uso de $\beta$-lactámicos, especialmente de cefalosporinas, como arsenal terapéutico. El predominio de ceftriaxona $\mathrm{u}$ otras cefalosporinas de $3^{\mathrm{a}}$ generación es un hecho observado en diferentes latitudes y escenarios, ocu- 


\begin{tabular}{|c|c|c|c|c|c|c|}
\hline Especie & Compuesto & $n^{*}$ & Promedio (\%) & Mínimo (\%) & Máximo (\%) & DE (\%) \\
\hline S. aureus & Cloxacilina & 7 & 42,5 & 28,6 & 92,6 & 22,7 \\
\hline S. pneumoniae & Penicilina & 5 & 89,5 & 73,7 & 100,0 & 9,9 \\
\hline Enterococcus sp & Vancomicina & 5 & 98,0 & 90,1 & 100,0 & 4,4 \\
\hline E. coli & Ceftazidima & 4 & 71,2 & 55,6 & 100,0 & 20,0 \\
\hline E. coli & Ciprofloxacina & 5 & 66,0 & 48,0 & 92,4 & 16,6 \\
\hline K. pneumoniae & Ceftazidima & 6 & 24,9 & 0,0 & 57,0 & 19,7 \\
\hline K. pneumoniae & Ciprofloxacina & 7 & 30,6 & 0,0 & 50,0 & 17,1 \\
\hline K. pneumoniae & Imipenem-cilastatina & 6 & 100,0 & 100,0 & 100,0 & 0,0 \\
\hline A. baumannii & Sulbactam/cefoperazona & 2 & 75,0 & 50,0 & 100,0 & 35,4 \\
\hline A. baumannii & Imipenem-cilastatina & 7 & 98,2 & 87,5 & 100,0 & 4,7 \\
\hline$P$. aeruginosa & Imipenem-cilastatina & 7 & 76,2 & 33,0 & 100,0 & 23,9 \\
\hline$P$. aeruginosa & Amikacina & 7 & 80,0 & 61,8 & 92,3 & 12,6 \\
\hline
\end{tabular}

pando el primer o segundo lugar en varias series. Por ejemplo, autores de Turquía reportan cifras de 8,84 DDD por 100 días camas para ceftriaxona en un estudio que abarcó 15 hospitales ${ }^{2}$; ceftriaxona y cefotaxima en conjunto ocuparon el primer o segundo lugar en un seguimiento de cinco años en un hospital de Carolina del Norte en E.U.A. ${ }^{1}$, fenómeno que también fue observado en Israel para ceftazidima y ceftriaxona ${ }^{19}$. El uso preferente de cefalosporinas de $3^{\mathrm{a}}$ generación puede explicarse por su inclusión en diversas guías, su flexibilidad terapéutica para lograr cobertura antimicrobiana sobre infecciones supra e infra-diafragmáticas, el bajo costo relativo para algunos componentes y su excelente perfil de seguridad. En el caso de cefazolina, su penetración obedece en parte a su utilización profiláctica en varias condiciones. El mercado de las cefalosporinas tiene un alcance mundial y se ha asociado a la emergencia de complejas resistencias hacia estos mismos compuestos en bacilos gramnegativos productores de BLEE ( $\beta$-lactamasas de espectro extendido), a la aparición de Enterococcus sp resistente a vancomicina o a un mayor riesgo de candiduria para citar algunos ejemplos $3,5,19,20$.

En tercer lugar, se logró identificar algunos factores que se asociaron significativamente a las variaciones observadas en el consumo de diferentes compuestos. En el análisis multivariado, se logró determinar que el consumo de cefazolina parece depender del uso permisivo de este compuesto y que el uso de ciprofloxacina y fluconazol ev están asociados a los hospitales que no están en la esfera pública.

Finalmente, fue posible encontrar correlaciones entre el consumo de ciertos compuestos y los porcentajes de susceptibilidad a algunas moléculas. Debido al
Tabla 8. Diferencias en los porcentajes de susceptibilidad ante antimicrobianos específicos según tipo de hospitales

\begin{tabular}{lccc} 
Especie y Compuesto & $\begin{array}{c}\text { \% susceptibilidad } \\
\pm D E\end{array}$ & $\begin{array}{c}\% \text { susceptibilidad } \\
\pm D E\end{array}$ & P \\
& $\begin{array}{c}\text { Hospitales públicos } \\
\text { Otros hospitales }\end{array}$ & \\
\hline Enterococcus sp S a vancomicina & $96,6 \pm 8,12$ & $92,3 \pm 9,8$ & $<0,05$ \\
\hline P. aeruginosa S a imipenem-cilastatina & $84,0 \pm 16,9$ & $65,0 \pm 16,8$ & $<0,05$ \\
\hline DE: desviación estándar & & &
\end{tabular}

\section{Tabla 9. Correlaciones entre susceptibilidad y consumos de antimicrobianos}

\begin{tabular}{lllcr} 
Consumo de & Especie & $\begin{array}{c}\text { Menor suscep- } \\
\text { tibilidad a }\end{array}$ & $\begin{array}{c}\text { Coeficiente } \\
\text { de } \\
\text { correlación }\end{array}$ & p \\
\hline Imipenem-cilastatina & P. aeruginosa & Imipenem-cilastatina & $-0,42$ & $<0,05$ \\
\hline Imipenem-cilastatina & A. baumannii & Imipenem-cilastatina & $-0,47$ & $<0,05$ \\
\hline Colistín & A. baumannii & Imipenem-cilastatina & $-0,66$ & 0,001 \\
Colistín & P. aeruginosa & Imipenem-cilastatina & $-0,45$ & $<0,05$ \\
\hline Linezolid & Enterococcus sp & Vancomicina & $-0,45$ & $<0,05$ \\
\hline
\end{tabular}

diseño transversal del estudio, no es posible establecer la causalidad de estas relaciones, aunque en el caso del mayor uso de colistín, es posible que esta preferencia probablemente obedezca a la emergencia de bacilos gramnegativos no fermentadores resistentes a imipenem-cilastatina en algunos hospitales. De la misma manera, el mayor uso de linezolid detectado en algunos sitios, obedece probablemente a la aparición de cepas de Enterococcus sp resistentes a vancomicina. Ambos fenómenos han sido reportados por autores nacionales ${ }^{21,22}$. Por otra parte, la asociación identifica- 
da entre un mayor consumo de imipenem-cilastatina y una menor susceptibilidad a este compuesto en bacilos gramnegativos no fermentadores, sugiere que la presión selectiva involucrada en su utilización es responsable de la emergencia de esta resistencia.

Los resultados globales de este trabajo tienen varias proyecciones incluyendo la necesidad de reforzar las medidas de control del uso de antimicrobianos en hospitales, especialmente en la esfera no pública, la capacitación de los médicos a nivel nacional $\mathrm{y}$, finalmente, la necesidad de supervisar el cumplimiento de la regulación vigente, algo que no se ha hecho en forma sistemática.

Este trabajo tiene varias limitaciones, entre ellas la inclusión de un número limitado de clínicas privadas ubicadas sólo en la RM y la falta de un seguimiento temporal en el consumo de los antimicrobianos. Además, los programas de control y restricción de antimicrobianos detectados en la encuesta no dan cuenta de la intensidad de su ejercicio, de los programas de capacitación asociados y desconocen el peso que pudo haber tenido la dotación variable de camas pediátricas o de pacientes con diálisis, a lo largo de los hospitales, factores que tergiversan estos consumos por la utilización de dosis más bajas para algunos compuestos. Finalmente, no se evaluó en este trabajo el uso de compuestos orales en los hospitales.

Agradecimientos. Los autores agradecen al Sr. QF Ariel Castro de la Unidad de Epidemiología Clínica del Hospital Clínico por su apoyo en el análisis estadístico.

\section{Resumen}

El consumo de antimicrobianos parenterales en hospitales y sus sistemas de control no han sido evalua- dos previamente en Chile. Para conocer la magnitud de este consumo (en DDD por 100 días camas), los potenciales factores asociados y los sistemas de regulación, se aplicó una encuesta en una muestra de hospitales públicos y privados a lo largo del país durante el año 2005. Un total de 29 hospitales o clínicas contestaron la encuesta, 20 de tipo público (69\%). Sólo 48,1\% declaró contar con un comité independiente de antimicrobianos y $17,2 \%$ permite el uso libre sin ninguna restricción. Los carbapenémicos y glicopéptidos fueron los compuestos más supervisados ( 82,8 y $75,9 \%$, respectivamente). Los programas de control sobre antimicrobianos fueron más frecuentes en los hospitales públicos que en el resto y sólo los hospitales no públicos permitían el uso libre sin restricciones. El consumo global de los compuestos antibacterianos alcanzó 59,98 DDD por 100 días camas, representando los $\beta$-lactámicos el 74,3\% de este consumo (44,57 DDD por 100) y las cefalosporinas el $43 \%$ de este valor (25,78 DDD por 100). El consumo de cloranfenicol, penicilina $\mathrm{G}$ y cloxacilina fue más elevado en los hospitales públicos. En contraste, el consumo de imipenemcilastatina, linezolid, cefuroxima y caspofungina, fue más elevado en los de tipo no público. El mayor consumo de cefazolina apareció ligado, en un análisis multivariado, al uso libre sin restricción y el mayor consumo de ciprofloxacina, a los hospitales no públicos. Aquellos lugares que tuvieron un mayor consumo de imipenem-cilastatina presentaron una menor susceptibilidad a este compuesto en cepas de $P$. aeruginosa y A. baumannii. El consumo de linezolid fue mayor en lugares con mayores porcentajes de aislados de Enterococcus sp resistente a vancomicina. Los datos demuestran que es necesario reforzar las medidas ministeriales vigentes desde 1999 sobre el uso racional de antimicrobianos en hospitales.

\section{Referencias}

1.- Cook P P, Catrou P G, Christie J D, Young P D, Polo R E. Reduction in broadspectrum antimicrobial use associated with no improvement in hospital antibiogram. J Antimicrob Chemother 2004; 53: 853-9.

2.- Hoşolu S, Esen S, Ozturk R, Altindis M, Ertek M, Kaygusuz S, et al. The effect of a restriction policy on the antimicrobial consumption in Turkey: a country-wide study. Eur J Clin Pharmacol 2005; 61: 727-31.

3.- Hsueh P R, Chen W H, Luh K T. Relationships between antimicrobial use and antimicrobial resistance in Gram-negative bacteria causing nosocomial infections from
1991-2003 at a university hospital in Taiwan. Int J Antimicrob Agents 2005; 26: 463-72.

4.- Janknegt R, Lashof A O, Gould I M, van der Meer J W M. Antibiotic use in Dutch hospitals, 1991-1996. J Antimicrob Chemother 2000; 45: 251-6.

5.- Kolar M, Urbanek K, Vagnerova I, Koukalova D. The influence of antibiotic use on the occurrence of vancomycinresistant enterococci. J Clin Pharm Ther 2006; 31: 67-72.

6.- Bavestrello L, Cabello A. Estudio del consumo de antimicrobianos en la comunidad. Chile, 10 años después. Rev Chil Infectol 1999; 16: 185-90.

7. - Bavestrello L, Cabello A, Casanova D.
Impacto de medidas reguladoras en las tendencias de consumo de antibióticos en Chile. Rev Méd Chile 2002; 130: 1265-72.

8. - Brieva J, Danhier A, Villegas G, Yates T, Pérez H. Modalidades del uso de antibióticos en Concepción, Chile. Bol Of Sanit Panam 1987; 103: 363-72.

9.- Lira G, Giglio M S, Zúñiga M, Pinto M E. Consumo de antimicrobianos y variación de resistencia en bacilos Gram negativos en un período de 10 años: Hospital San Juan de Dios, Chile. Rev Chil Infectol 1999; 16: 199-210.

10.- Boccia D, Alegiani S S, Pantosti A, Moro M L, Traversa G. The geographical relationship between the use of antimicrobial drugs and the pattern of resistance for 
Streptococcus pneumoniae in Italy. Eur J Clin Pharmacol 2004; 60: 115-9.

11.- Pérez Aldana L, Kato M, Nakagawa S, Kawasaraki M, Nagasako T, Mizushima T, et al. The relationship between consumption of antimicrobial agents and the prevalence of primary Helicobacter pylori resistance. Helicobacter 2002; 7: 306-9.

12.- MacKenzie F M, Bruce J, Struelens M J, Goossens H, Mollison J, Gould I M, et al. Antimicrobial drug use and infection control practices associated with the prevalence of methicillin-resistant Staphylococcus aureus in European hospitals. Clin Microbiol Infect 2007; 13: 269-76.

13.- Mohr J F, Jones A, Ostrosky-Zeichner L, Wanger A, Tillotson G. Associations between antibiotic use and changes in susceptibility patterns of Pseudomonas aeruginosa in a private, university-affiliated teaching hospital: an 8-year-experience: 1995-2002. Int J Antimicrob Agents 2004; 24: 346-51.

14.- Monsen T, Rönnmark M, Olofsson C, Wiström J. Antibiotic susceptibility of staphylococci isolated in blood cultures in relation to antibiotic consumption in hospital wards. Scand J Infect Dis 1999; 31: 399-404.

15.- Mutnick A H, Rhomberg P R, Sader H S, Jones R N. Antimicrobial usage and resistance trend relationship form the MYSTIC Programme in North America (1999-2001). J Antimicrob Chemother 2004; 53: 290-6.

16.- Rogues A M, Dumartin C, Amadeo B, Venier A G, Marty N, Parneix P, et al. Relationship between rates of antimicrobial consumption and the incidence of antimicrobial resistance in Staphylococcus aureus and Pseudomonas aeruginosa isolates from 47 French hospitals. Infect Control Hosp Epidemiol 2007; 28: 1389-95.

17.- Riedel S, Beekman S E, Heilmann K P, Richter S S, García de Lomas J, Ferech M, et al. Antimicrobial use in Europe and antimicrobial resistance in Streptococcus pneumoniae. Eur J Clin Microbiol Infect Dis 2007; 26: 485-90.
18.- World Health Organization. WHO Collaborating Centre for Drug Statistics and Methodology. http://www.whocc.no/atcddd (accedido agosto del 2007)

19. - Weinberger M, Sweet S, Leibovici L, Pitlik S D, Samra Z. Correlation between candiduria and departmental antibiotic use J Hosp Infect 2003; 53: 183-6.

20. - Vander Stichele R H, Elseviers M M, Ferech M, Blot S, Goznes H. Hospital consumption of antibiotics in 15 European countries: results of the ESAC retrospective data collection (1997-2002). J Antimicrob Chemother 2006; 58: 159-67.

21.- Fica A, Céspedes J, Gompertz M, Jalon M, Sakurada A, Sáez E. Colistín en infecciones nosocomiales por bacilos gramnegativos pan-resistentes. Rev Chil Infectol 2007; 24: 360-7

22.- Fica A, Jemenao M I, Bilbao P, Ruiz G, Sakurada A, Pérez de Arce E, et al. Emergencia de infecciones por Enterococcus sp resistente a vancomicina en un hospital universitario en Chile. Rev Chil Infectol 2007; 24: 462-71. 